\title{
Efficacy and Safety of Runzao Zhiyang Capsule as an Add-On Therapy for Chronic Eczema: A Systematic Review and Meta-Analysis
}

\author{
Ming Li (D), Boyang Zhou (D), Lihong Zhou $(\mathbb{D}$, and Linfeng Li (i) \\ Department of Dermatology, Beijing Friendship Hospital, Capital Medical University, Beijing, China \\ Correspondence should be addressed to Linfeng Li; zoonli@sina.com
}

Received 8 October 2020; Revised 2 March 2021; Accepted 5 March 2021; Published 17 March 2021

Academic Editor: Mohammad Hashem Hashempur

Copyright ( 2021 Ming Li et al. This is an open access article distributed under the Creative Commons Attribution License, which permits unrestricted use, distribution, and reproduction in any medium, provided the original work is properly cited.

\begin{abstract}
Background. Runzao Zhiyang capsule (RZC), an oral Chinese herbal medicine, has been widely used for chronic eczema in China for many years. This study aims to evaluate the efficacy and safety of RZC as an add-on therapy to conventional treatment for chronic eczema. Methods. Randomized controlled trials (RCTs) assessing the efficacy and safety of RZC as an add-on therapy for chronic eczema were retrieved from eight literature databases from their inception to 31 August, 2020, including CNKI, WanFang, VIP, Sinomed, PubMed, Cochrane Library, Web of Science, and Embase. The Cochrane risk of bias tool was used to assess the methodological quality of the included studies. The data were analyzed by RevMan5.3 software. Results. A total of 18 RCTs involving 1896 patients with chronic eczema were included. Compared with no oral treatment, RZC was superior on the total efficacy rate (TER) $(\mathrm{RR}=1.45,95 \% \mathrm{CI}: 1.23$ to $1.72, P<0.0001)$, Eczema Area and Severity Index (EASI) $(\mathrm{MD}=-0.73,95 \% \mathrm{CI}$ : -0.90 to $-0.56, P<0.00001$ ), and Visual Analogue Scale (VAS) for pruritus (MD $=-2.76,95 \% \mathrm{CI}:-4.53$ to $-0.99, P=0.002$ ). Similar results were also seen in a randomized, placebo-controlled trial. Compared with the antihistamine (AH) group, TER in the RZC combined with AH group was significantly higher ( $R R=1.32,95 \%$ CI: 1.21 to $1.43, P<0.00001$ ), and the EASI score $(\mathrm{MD}=-0.29,95 \% \mathrm{CI}:-0.38$ to $-0.20, P<0.001)$, the VAS score $(\mathrm{MD}=-0.19,95 \% \mathrm{CI}:-0.23$ to $-0.15, P<0.00001)$, and the level of serum total $\operatorname{IgE}(\mathrm{MD}=-9.83 \mathrm{ng} / \mathrm{ml}, 95 \% \mathrm{CI}$ : -11.66 to $-8.00 \mathrm{ng} / \mathrm{ml}, P<0.00001)$ decreased more significantly in the RZC combined with AH group. In terms of safety, mild gastrointestinal diseases occurred more frequently in the RZC group, and no serious adverse effect was reported. Conclusions. RZC as an add-on therapy to conventional treatment shows good effects on chronic eczema, and there is no severe side effect from short-term use of RZC. However, due to suboptimal quality of the included studies, more large-sample and high-quality RCTs are needed to improve the evidence quality.
\end{abstract}

\section{Introduction}

Eczema, also known as "atopic dermatitis" or "neurodermatitis" in many countries, is a common skin disease characterized by a wide spectrum of skin lesions and pruritus $[1,2]$. It affects $15 \%$ to $20 \%$ of children and $1 \%$ to $3 \%$ of adults around the world [3]. In China, the prevalence of eczema in children is estimated to be $8.3 \%$ to $12.94 \%[4,5]$. The pathogenesis of eczema has not been fully understood, and it is associated with genetic and environmental factors. Although not life threatening, eczema can lead to sleeplessness and have a substantial impact on quality of life. Patients with eczema are more likely to suffer from anxiety and depression $[6,7]$, resulting in serious public health problems and economic costs [8].

Currently, topical treatments are the mainstay for the treatment of mild to moderate eczema. Moisturizers are widely applied to restore epidermal barrier function. Topical corticosteroids (TCSs) and topical calcineurin inhibitors (TCIs) could reduce lesions and relieve pruritus by inhibiting skin inflammation. Topical antiseptics showed antimicrobial activity against Staphylococcus aureus, which is predominantly associated with flares of eczema. Moreover, antihistamines (AHs) are recommended to be used as a systemic add-on treatment to relieve pruritus $[2,9]$. However, the side effects of $\mathrm{AH}$, such as drowsiness and fatigue, 
may impair concentration and reduce productivity. Moreover, some patients with chronic eczema are not satisfied with the effect of $\mathrm{AH}$ combined with topical medicine. In order to avoid adverse effects and to attain better clinical effects, many patients with different dermatological diseases, including chronic eczema, have chosen to use complementary and alternative medicines for the treatment of eczema, such as Chinese herbal medicine (CHM) [10-12].

In China, Runzao Zhiyang capsule (RZC), an oral CHM, has been widely used to treat chronic eczema for many years. It is a mixture of six Chinese herbs, including Polygoni Multiflori Radix, Polygoni Multiflori Radix Praeparata, Rehmanniae Radix, Mori folium, Sophorae flavescentis Radix, and Urtica dentata Hand. The details of RZC are provided in Table 1. A lot of modern pharmacological studies have proved the effects of these components. Polygoni Multiflori Radix shows anti-inflammatory activity by decreasing Th2 cytokine levels, such as interleukin- (IL-) 4, IL-5, and IL-13, and inhibiting the mRNA expression of GATA-3 [13]. Rehmanniae Radix could inhibit the mRNA expression of IL-4 and tumor necrosis factor- (TNF-) $\alpha$ in lesions and suppress mast cell activations, such as histamine release and production of IL- $1 \beta$ and IL-6 [14, 15]. Mori folium shows moderate antibacterial activity against Staphylococcus aureus and inhibits NF- $\kappa \mathrm{B}$-mediated inflammatory response, such as IL-1 $\beta$ and IL-6 [16, 17]. Sophorae flavescentis Radix not only inhibits the mast cellmediated histamine release and decreases the levels of interferon- (IFN-) $\gamma$ and TNF- $\alpha$ in lesions but also significantly inhibits the 5-hydroxytryptamine-induced scratching $[18,19]$. Total coumarin, an extraction isolated from Urtica dentata Hand, increases the production of IL-10 and transforming growth factor- (TGF-) $\beta$ in dendritic cells and induces the generation of $\mathrm{CD} 4{ }^{+} \mathrm{CD} 25^{+}$Treg cells [20]. Some clinical trials have reported that RZC combined with biomedicine could reduce skin lesions and relieve pruritus for patients with chronic eczema [21, 22].

Although many randomized controlled trials (RCTs) on RZC as an add-on treatment for chronic eczema have been conducted, there is no systematic review and meta-analysis to integrate these RCTs. Therefore, this study aims to systematically evaluate the efficacy and safety of RZC as an addon therapy to conventional treatment for the treatment of chronic eczema.

\section{Methods}

This article was conducted in accordance with the Preferred Reporting Items for Systematic Reviews and Meta-Analyses (PRISMA) statement [23]. The PRISMA checklist is presented in Supplementary Table 1.

2.1. Literature Search. The four English databases and four Chinese databases were searched from their inception to 31 August, 2020, including PubMed, Cochrane Library, Web of Science, Embase, China National Knowledge Infrastructure (CNKI, https://www.cnki.net/), WanFang Database (WanFang, http://new.wanfangdata.com.cn/), Chinese Scientific
Journal Database (VIP, http://qikan.cqvip.com/), and Chinese Biomedical Database (Sinomed, http://www.sinomed. ac.cn/). The search strategy was as follows: (("eczema" [MeSH] OR "eczema" [Title/Abstract]) OR ("dermatitis" [MeSH] OR "dermatitis" [Title/Abstract])) AND ("Runzao Zhiyang capsule" [Title/Abstract] OR "Runzaozhiyang capsule" [Title/Abstract] OR "Run Zao Zhi Yang capsule" [Title/Abstract]). In addition, the reference lists of relevant studies were also searched to identify other potentially eligible studies. The research was conducted by two independent authors (ML and BYZ), and any disagreements were resolved by the third author (LHZ).

2.2. Inclusion and Exclusion Criteria. The eligible studies were selected based on the following inclusion criteria: (1) RCTs published in English or Chinese; (2) participants were diagnosed with chronic eczema by dermatologists, regardless of age, gender, and disease duration; (3) the experimental group was treated with RZC or RZC combined with $\mathrm{AH}$, while the control group was treated with no oral treatment, placebo, or AH. All participants were treated with the same topical medicine, including moisturizers, TCSs, TCIs, and topical antiseptics. (4) The primary outcomes included total efficacy rate (TER) and Eczema Area and Severity Index (EASI). TER is the proportion of participants with the improvement of symptoms and signs $\geq 60 \%$ of baseline at the end of treatment, and EASI is an international tool to assess overall severity of skin lesion [24]. The secondary outcomes included severity of pruritus, serum total IgE level, and adverse events. The Visual Analogue Scale (VAS) is used to assess the severity of pruritus [25].

Animal experiments, case reports, reviews, duplicate studies, inappropriate interventions, and unavailable studies were excluded.

2.3. Study Selection and Data Extraction. Based on the inclusion and exclusion criteria, two authors (ML and BYZ) independently screened the titles and abstracts to identify potentially eligible studies and read full texts to determine the final included studies. The disagreement was settled by the third author (LHZ). The extracted data included the first author, publication year, the number of participants in each group, gender, age, interventions, and outcomes.

2.4. Quality Assessment. The methodological quality of the included studies was evaluated using the Cochrane risk of bias tool [26]. It contains seven items, including random sequence generation, allocation concealment, blinding of participants and personnel, blinding of outcome assessment, incomplete outcome data, selective reporting, and other bias. The baselines of disease severity between two groups were considered as the source of other bias. Quality of each item was divided into high, unclear, and low risk of bias. Two authors (ML and BYZ) independently conducted the assessment, and any disagreements were tackled by the third author (LHZ). 
TABLE 1: Ingredients of Runzao Zhiyang capsule*.

\begin{tabular}{|c|c|c|c|c|}
\hline Herb & $\begin{array}{l}\text { The part of } \\
\text { the plant }\end{array}$ & $\begin{array}{l}\text { Preparation } \\
\text { method }\end{array}$ & Chinese name & $\begin{array}{l}\text { The name from Chinese } \\
\text { Pharmacopoeia }\end{array}$ \\
\hline Reynoutria multiflora (Thunb.) Moldenke & Root & Water extract & He-Shou-Wu (何首乌) & Polygoni multiflori Radix \\
\hline Reynoutria multiflora (Thunb.) Moldenke & Root & Powder & $\begin{array}{l}\text { Zhi-He-Shou-Wu } \\
\text { (制何首乌) }\end{array}$ & Polygoni multiflori Radix Praeparata \\
\hline Rehmannia glutinosa (Gaertn.) DC & Root & Water extract & Sheng-Di-Huang (生地黄) & Rehmanniae Radix \\
\hline Morus alba L. & Leaf & Water extract & Sang-Ye (桑叶) & Mori folium \\
\hline Sophora flavescens Aiton & Root & Water extract & Ku-Shen (苦参) & Sophorae flavescentis Radix \\
\hline Laportea bulbifera (Sieb. et Zucc.) Wedd. & Root & Water extract & Hong-Huo-Ma (红活麻) & Urtica dentata Hand \\
\hline
\end{tabular}

*Each RZC is $500 \mathrm{mg}$ and contains $85 \mathrm{mg}$ of Polygoni multiflori Radix, $77 \mathrm{mg}$ of Polygoni multiflori Radix Praeparata, $125 \mathrm{mg}$ of Rehmanniae Radix, $85 \mathrm{mg}$ of Mori folium, $85 \mathrm{mg}$ of Sophorae flavescentis Radix, and $43 \mathrm{mg}$ of Urtica dentata Hand.

2.5. Statistical Analysis. RevMan5.3 software was used for data analysis. Dichotomous variables were expressed as risk ratio (RR) with $95 \%$ confidence interval (CI), and continuous variables were expressed as mean difference (MD) with 95\% CI. Heterogeneity between studies was measured by the chi-square test. A fixed-effect model was applied when $I^{2}<50 \%$ and $P>0.10$, and a random-effect model was used if not. If ten or more studies were pooled, a funnel plot was used to assess the potential publication bias. Significant differences were accepted when the $P$ value $<0.05$.

\section{Results}

3.1. Search Results. A total of 415 studies were identified from eight literature databases, of which 277 duplicates were excluded. 92 studies were excluded based on the titles and abstracts. After screening full texts, 28 studies were excluded further for various reasons. In the end, 18 studies [27-44] met the eligible criteria and were included in this metaanalysis. Figure 1 shows the flowchart of study selection.

3.2. Characteristics of Included Studies. All 18 studies involving 1896 patients were conducted in China. One study published in English was a multicenter clinical trial [33], while the rest were published in Chinese and conducted in a single center. The age of participants ranged from 14 to 79 years old, and the duration range of the treatment was 2 to 8 weeks. Of the included studies, six compared RZC to no oral treatment [27-32], one compared RZC to placebo [33], and eleven compared RZC combined with AH to AH [34-44]. The most frequently used outcome was adverse events, followed by TER, EASI, severity of pruritus, and serum total IgE level. The main characteristics of each study are presented in Table 2.

3.3. Quality Evaluation of Included Studies. Eight studies used random number tables or computer to generate random sequence; therefore, the risk of random sequence generation of them was low [27, 30, 31, 33, 34, 37, 39, 40]. Due to the lack of detailed information, the risk of random sequence generation of the remaining 10 studies was unclear. All studies failed to mention the methods of allocation concealment. Only one study took measures in blinding patients, healthcare providers, and outcome assessors [33]. All studies provided complete outcome data and reported predetermined outcome indicators. Due to the comparability of baselines of disease severity in two groups, other bias was evaluated to be low risk in eleven studies $[27,28,30-34,37,39,40,44]$. The rest did not report baseline comparability and were considered to be unclear risk. Figure 2 shows the risk of bias summary of included studies.

\subsection{Outcomes}

3.4.1. Total Efficacy Rate. Three studies reported the TER between the RZC group and the no oral treatment group. No significant heterogeneity was found $\left(I^{2}=0 \%, P=0.68\right)$, and a fixed-effect model was used. The pooled results showed that RZC was superior to no oral treatment on the TER $(\mathrm{RR}=1.45,95 \% \mathrm{CI}: 1.23$ to $1.72, P<0.0001)[28,30,32]$ (Figure 3).

One randomized, placebo-controlled trial investigated the effect of RZC on chronic eczema. The result revealed that the RZC group had a significantly higher TER compared with the placebo group after 4 weeks of treatment $(R R=4.08$, 95\% CI: 2.69 to $6.19, P<0.00001$ ) [33] (Figure 3).

Eight studies, which compared RZC combined with AH to $\mathrm{AH}$, used TER as an outcome indicator. Due to low statistical heterogeneity $\left(I^{2}=28 \%, P=0.20\right)$, a fixed-effect model was applied. The pooled results showed that the TER of the RZC combined with $\mathrm{AH}$ group was significantly higher than that of the AH group ( $\mathrm{RR}=1.32,95 \% \mathrm{CI}: 1.21$ to $1.43, P<0.00001)[34-36,38-41,43]$ (Figure 3).

3.4.2. EASI. One study used EASI to compare the effects between RZC and no oral treatment. The result indicated that RZC was more effective than no oral treatment in terms of reducing EASI score after 4 weeks of treatment $(\mathrm{MD}=-0.73$, 95\% CI: -0.90 to $-0.56, P<0.00001$ ) [27] (Figure 4 ).

One randomized, placebo-controlled trial found that the EASI score of the RZC group was significantly lower than that of the placebo group after 4 weeks of treatment $(\mathrm{MD}=-1.60$, 95\% CI: -2.46 to $-0.74, P=0.0003$ ) [33] (Figure 4).

EASI score of the RZC combined with AH group and the AH group was evaluated in two studies [34, 40]. Due to no significant heterogeneity $\left(I^{2}=0 \%, P=1.00\right)$, a fixed-effect model was used. The pooled results showed that the EASI score of the RZC combined with AH group could be significantly reduced in comparison with the $\mathrm{AH}$ group 


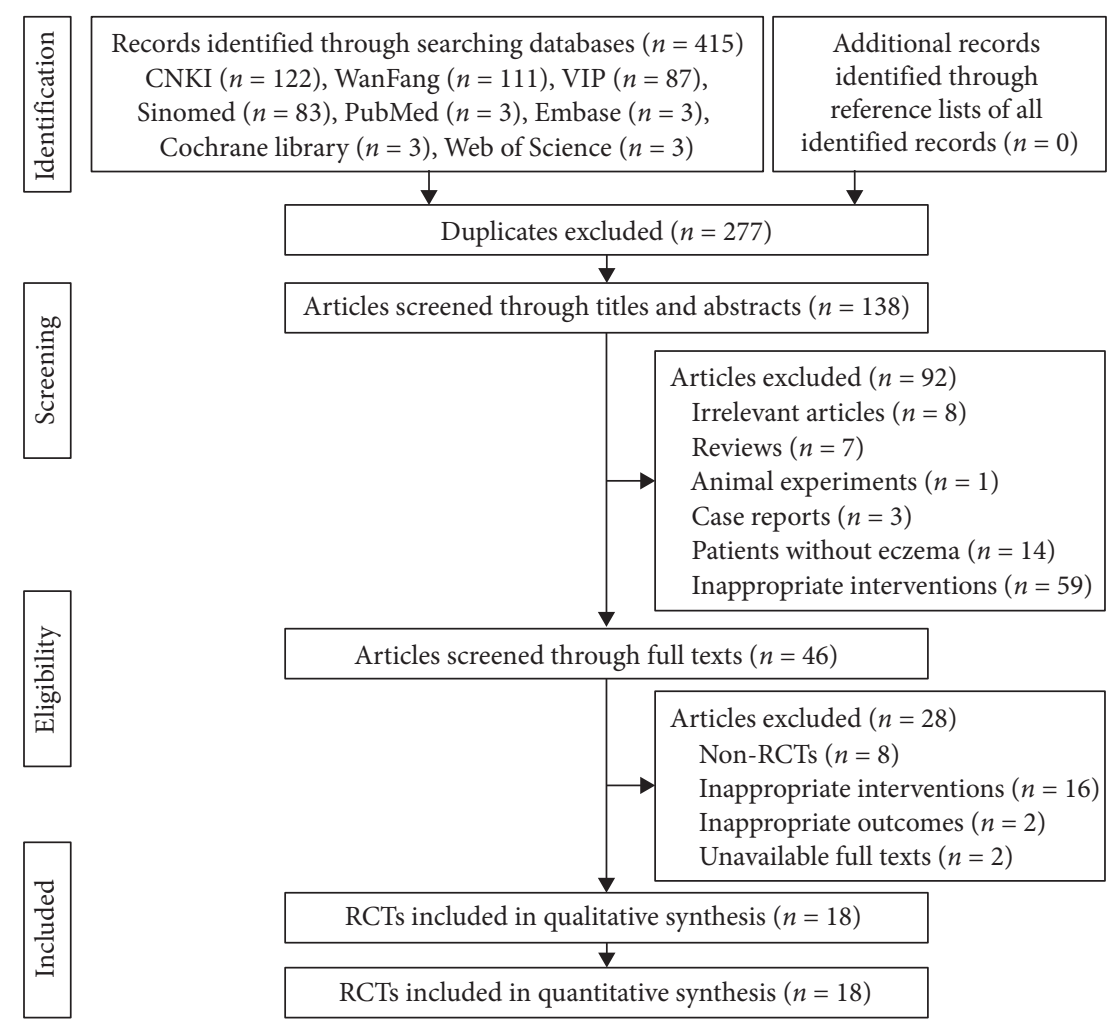

FIgURE 1: The flowchart of study selection.

$(\mathrm{MD}=-0.29, \quad 95 \% \quad \mathrm{CI}: \quad-0.38$ to $-0.20, \quad P<0.00001)$ (Figure 4).

3.4.3. Severity of Pruritus. One study, which compared RZC with no oral treatment, found that VAS score of the RZC group was significantly lower than that of the control group after 4 weeks of treatment $(\mathrm{MD}=-2.76,95 \% \mathrm{CI}:-4.53$ to -0.99, $P=0.002$ ) [30] (Figure 5).

One randomized, placebo-controlled trial used VAS to evaluate the severity of pruritus during 4 weeks of treatment, and the result revealed that RZC could relieve pruritus in comparison to the placebo $(\mathrm{MD}=-10.77,95 \% \mathrm{CI}:-17.02$ to -4.52, $P=0.0007$ ) [33] (Figure 5).

VAS score of the RZC combined with AH group and the $\mathrm{AH}$ group was measured in two studies [34, 40]. There was no significant heterogeneity between them $\left(I^{2}=0 \%\right.$, $P=1.00$ ), and a fixed-effect model was used. The pooled results showed that RZC combined with $\mathrm{AH}$ was superior to $\mathrm{AH}$ alone in alleviating pruritus $(\mathrm{MD}=-0.19,95 \% \mathrm{CI}:-0.23$ to $-0.15, P<0.00001$ ) (Figure 5).

3.4.4. Serum Total IgE Level. Serum total IgE level was reported in four studies which compared RZC combined with $\mathrm{AH}$ to $\mathrm{AH}$ alone $[34,35,37,40]$. Low heterogeneity was observed $\left(I^{2}=43 \%, P=0.15\right)$, and a fixed-effect model was used. The pooled results showed that the serum total IgE level of the RZC combined with AH group was significantly lower than that of the AH group $(\mathrm{MD}=-9.83 \mathrm{ng} / \mathrm{ml}, 95 \%$ CI: -11.66 to $-8.00 \mathrm{ng} / \mathrm{ml}, P<0.00001$ ) (Figure 6).
3.4.5. Adverse Events. In total, 14 studies mentioned adverse events during the treatment [27-33, 38-44]. In terms of gastrointestinal adverse events, few patients in the RZC group experienced gastrointestinal diseases, such as mild diarrhea and gastrointestinal discomfort. Six studies reported gastrointestinal adverse events in the RZC group and the no oral treatment group [27-32]. No significant heterogeneity was found $\left(I^{2}=0 \%, P=0.82\right)$, and a fixed-effect model was used. The pooled results showed that the incidence of gastrointestinal adverse events of the RZC group was significantly higher than that of the no treatment group $(\mathrm{RR}=3.93,95 \% \mathrm{CI}: 1.28$ to $12.09, P=0.02$ ) (Figure 7$)$. The same result was also seen in the seven studies comparing RZC combined with $\mathrm{AH}$ to $\mathrm{AH}$ lone [38-44]. Due to low heterogeneity $\left(I^{2}=17 \%, P=0.31\right)$, a fixed-effect model was used. The pooled results showed that RZC combined with $\mathrm{AH}$ significantly increased the incidence of gastrointestinal diseases compared with $\mathrm{AH}$ alone $(\mathrm{RR}=3.00,95 \% \mathrm{CI}: 1.10$ to $8.18, P=0.03$ ) (Figure 7). However, the randomized, placebo-controlled trial found that 13 patients in the RZC group and 11 patients in the placebo group suffered from gastrointestinal diseases, and the incidences between two groups were not statistically different $(\mathrm{RR}=1.17,95 \% \mathrm{CI}$ : 0.55 to $2.51, P=0.68$ ) (Figure 7 ) [33].

In terms of laboratory examinations, six studies monitored blood routine, urine routine, liver function, and renal function during the 2 to 8 weeks of treatment. Five out of the six studies reported that 293 patients in the RZC group had normal results of these laboratory examinations $[30,39,40,43,44]$. In the randomized, placebo-controlled 
TABLE 2: The characteristics of 18 included studies.

\begin{tabular}{|c|c|c|c|c|c|c|c|c|}
\hline \multirow[t]{2}{*}{ Study ID } & \multicolumn{2}{|c|}{$\begin{array}{l}\text { Sample size (male/ } \\
\text { female) }\end{array}$} & \multicolumn{2}{|c|}{ Age (years) } & \multicolumn{2}{|r|}{ Interventions } & \multirow{2}{*}{$\begin{array}{c}\text { Treatment } \\
\text { duration } \\
\text { (weeks) }\end{array}$} & \multirow[t]{2}{*}{ Outcomes } \\
\hline & E & $\mathrm{C}$ & $\mathrm{E}$ & $\mathrm{C}$ & $\mathrm{E}$ & $\mathrm{C}$ & & \\
\hline $\begin{array}{l}\mathrm{Xu} \text { and Wang } \\
{[27]}\end{array}$ & $50(28 / 22)$ & $50(29 / 21)$ & $27-61$ & $27-60$ & $\mathrm{RZC}^{\#}+\mathrm{IC}$ & $\begin{array}{c}\text { Hydrocortisone butyrate } \\
\text { ointment }\end{array}$ & 4 & (2) (5) \\
\hline $\begin{array}{l}\text { Huang et al. } \\
\text { [33] }\end{array}$ & $\begin{array}{c}120 \\
(58 / 62)\end{array}$ & $118(51 / 67)$ & $18-70$ & $19-69$ & $\begin{array}{c}\mathrm{RZC}^{\#}+\text { urea } \\
\text { ointment }\end{array}$ & Placebo $^{\#}+$ urea ointment & 4 & (1) (2) (3) (5) \\
\hline $\begin{array}{l}\text { Zhang et al. } \\
\text { [34] }\end{array}$ & $73(37 / 36)$ & $73(42 / 31)$ & $22-35$ & $23-34$ & $\mathrm{RZC}^{\#}+\mathrm{IC}$ & $\begin{array}{c}\text { Epinastine } \\
\text { capsule + hydrocortisone } \\
\text { butyrate ointment } \\
\text { Eninastine }\end{array}$ & 4 & (1) (2) (3) (4) \\
\hline Jin [35] & 25 & 25 & $19-38$ & $19-38$ & $\mathrm{RZC}^{\#}+\mathrm{IC}$ & $\begin{array}{c}\text { Epinastine } \\
\text { capsule + hydrocortisone } \\
\text { butyrate ointment }\end{array}$ & 4 & (1) (4) \\
\hline Yuan [36] & $43(23 / 20)$ & $42(20 / 22)$ & $35-65$ & $36-66$ & $\mathrm{RZC}^{\#}+\mathrm{IC}$ & $\begin{array}{c}\text { Desloratadine tablet }+ \text { urea } \\
\text { ointment }\end{array}$ & 4 & (1) \\
\hline Chang [37] & $35(16 / 19)$ & $35(17 / 18)$ & $18-57$ & $19-58$ & $\mathrm{RZC}^{\#}+\mathrm{IC}$ & $\begin{array}{c}\text { Epinastine } \\
\text { capsule + hydrocortisone } \\
\text { butyrate ointment }\end{array}$ & 4 & (4) \\
\hline Shao [38] & $72(35 / 37)$ & $72(32 / 40)$ & $17-66$ & $18-65$ & $\mathrm{RZC}^{\#}+\mathrm{IC}$ & $\begin{array}{l}\text { Levocetirizine tablet }+ \\
\text { halcinonide ointment }\end{array}$ & 8 & (1)(5) \\
\hline Guan [39] & $49(24 / 25)$ & $49(22 / 27)$ & $19-57$ & $20-58$ & $\mathrm{RZC}^{\#}+\mathrm{IC}$ & $\begin{array}{l}\text { Mizolastine tablet }+ \\
\text { halometasone cream } \\
\text { Epinastine }\end{array}$ & 2 & (1) (5) \\
\hline Song et al. [40] & $72(38 / 34)$ & $72(40 / 32)$ & $23-58$ & $21-59$ & $\mathrm{RZC}^{\#}+\mathrm{IC}$ & $\begin{array}{l}\text { capsule + hydrocortisone } \\
\text { butyrate ointment } \\
\text { Epinastine }\end{array}$ & 4 & (1) (2) (3) (4) (5) \\
\hline Wang [41] & $30(15 / 15)$ & $30(14 / 16)$ & $18-60$ & $18-60$ & $\mathrm{RZC}^{\#}+\mathrm{IC}$ & $\begin{array}{l}\text { capsule + hydrocortisone } \\
\text { butyrate ointment }\end{array}$ & 4 & (1) (5) \\
\hline $\begin{array}{l}\text { Huang et al. } \\
{[42]}\end{array}$ & 30 & 30 & $21-53$ & $21-53$ & $\mathrm{RZC}^{\#}+\mathrm{IC}$ & $\begin{array}{l}\text { Levocetirizine } \\
\text { tablet }+ \text { hydrocortisone } \\
\text { butyrate cream }\end{array}$ & 8 & (5) \\
\hline Liu [43] & $78(42 / 36)$ & $78(44 / 34)$ & $14-58$ & $16-60$ & $\mathrm{RZC}^{\#}+\mathrm{IC}$ & $\begin{array}{c}\text { Levocetirizine } \\
\text { tablet }+ \text { hydrocortisone } \\
\text { ointment }\end{array}$ & 8 & (1) (5) \\
\hline Song et al. [44] & $62(37 / 25)$ & $60(32 / 28)$ & $18-60$ & $18-60$ & $\mathrm{RZC}^{\#}+\mathrm{IC}$ & $\begin{array}{c}\text { Mizolastine } \\
\text { tablet }+ \text { Halometasone } \\
\text { cream }\end{array}$ & 2 & (5) \\
\hline $\begin{array}{l}\text { Ruan and } \\
\text { Wang [28] }\end{array}$ & 34 & 33 & $18-34$ & $18-34$ & $\mathrm{RZC}^{\#}+\mathrm{IC}$ & $\begin{array}{l}\text { Mometasone } \\
\text { furoate cream }\end{array}$ & 4 & (1) (5) \\
\hline $\begin{array}{l}\text { Liu and Shen } \\
\text { [29] }\end{array}$ & $50(32 / 18)$ & $50(30 / 20)$ & $18-60$ & $18-60$ & $\mathrm{RZC}^{\#}+\mathrm{IC}$ & $\begin{array}{c}\text { Dexamethasone } \\
\text { ointment }\end{array}$ & 4 & (5) \\
\hline Zhu et al. [30] & $32(21 / 11)$ & $21(14 / 7)$ & $18-46$ & $18-47$ & $\mathrm{RZC}^{\#}+\mathrm{IC}$ & $\begin{array}{c}\text { Vaseline cream }+ \text { Mupirocin } \\
\text { ointment }\end{array}$ & 4 & (1) (3) (5) \\
\hline Zhou et al. [31] & 40 & 15 & $18-70$ & $18-70$ & $\mathrm{RZC}^{\#}+\mathrm{IC}$ & $\begin{array}{l}\text { Hydrocortisone and } \\
\text { urea cream }\end{array}$ & 4 & (5) \\
\hline Liu and $\mathrm{Li}$ [32] & 74 & 74 & $19-79$ & $19-79$ & $\mathrm{RZC}^{\#}+\mathrm{IC}$ & $\begin{array}{l}\text { Triamcinolone acetonide } \\
\text { and urea ointment }\end{array}$ & 3 & (1) (5) \\
\hline
\end{tabular}

E: experimental group; C: control group; RZC: Runzao Zhiyang capsule; IC: intervention of the control group. ${ }^{*} 4$ capsules each time and three times a day. (1) Total efficacy rate; (2) Eczema Area and Severity Index; (3) severity of pruritus; (4) serum total IgE level; (5) adverse events.

trial, 7 patients $(7 / 120,5.83 \%)$ in the RZC group and 11 patients $(11 / 119,9.24 \%)$ in the placebo group experienced a slight increase of liver transaminases, respectively, and the incidences of abnormal liver function between two groups were not significantly different $(\mathrm{RR}=0.63,95 \% \mathrm{CI}: 0.25$ to 1.57, $P=0.32$ ) [33].

3.5. Publication Bias. Because there were less than 10 studies in each comparison, the funnel plot was not used to assess the publication bias of the included studies.

\section{Discussion}

To the best of our knowledge, this is the first meta-analysis to assess the efficacy and safety of RZC as an add-on therapy for chronic eczema. The current study showed that the RZC group had a higher TER and a lower EASI score in comparison with the no oral treatment group and the placebo group. The pathogenesis in eczema is complex, and many different cytokines are involved, such as IL-4, IL-5, IL-13, and IL-17. Because of multiple ingredients with 


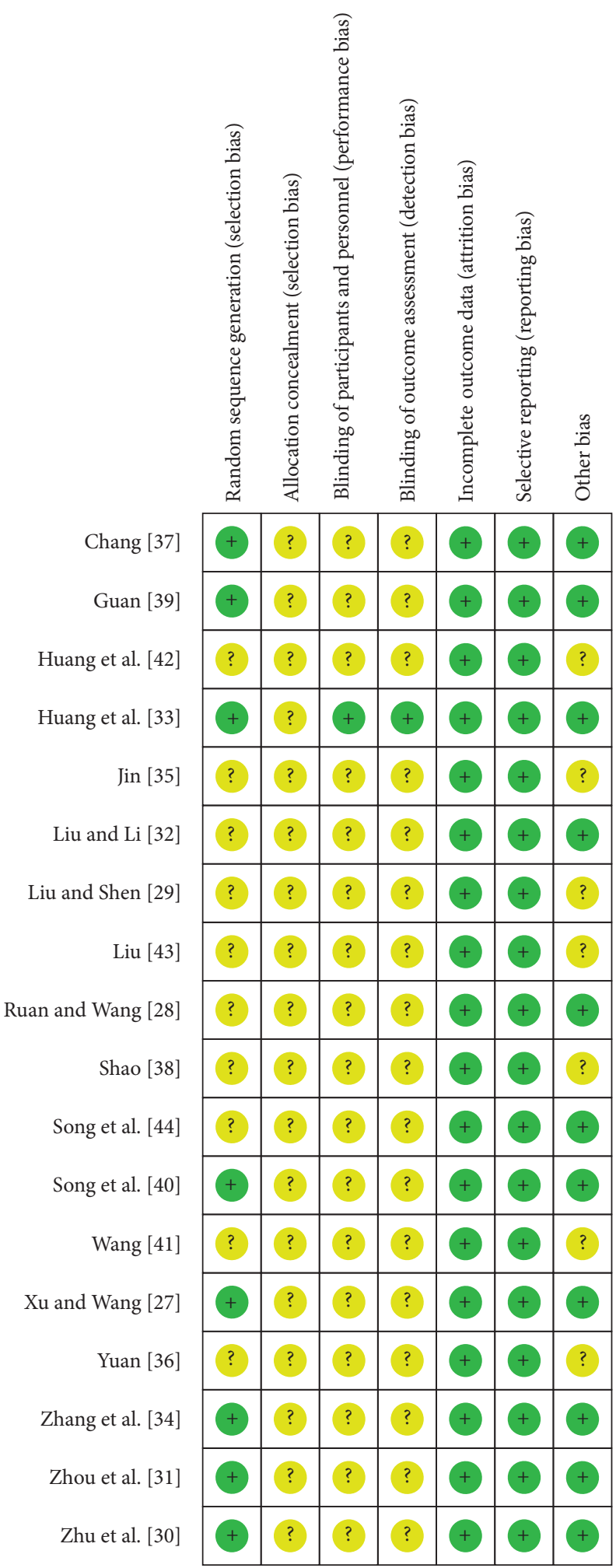

Figure 2: The risk of bias summary of included studies.

immune regulation function, RZC could possibly treat chronic eczema by multiple mechanisms. Some clinical trials have proved that RZC could rapidly decrease the levels of IL- 1 , IL-4, and TNF- $\alpha$ in serum $[45,46]$. One study on rats shows that RZC could regulate inflammation by decreasing the levels of IL- 6 and IL-17 and increasing the level of IL-10 in serum [47]. Therefore, RZC has a good effect on the treatment of chronic eczema and is effective as an add-on treatment to topical medicine. In addition, the current study also found that compared with AH, RZC 


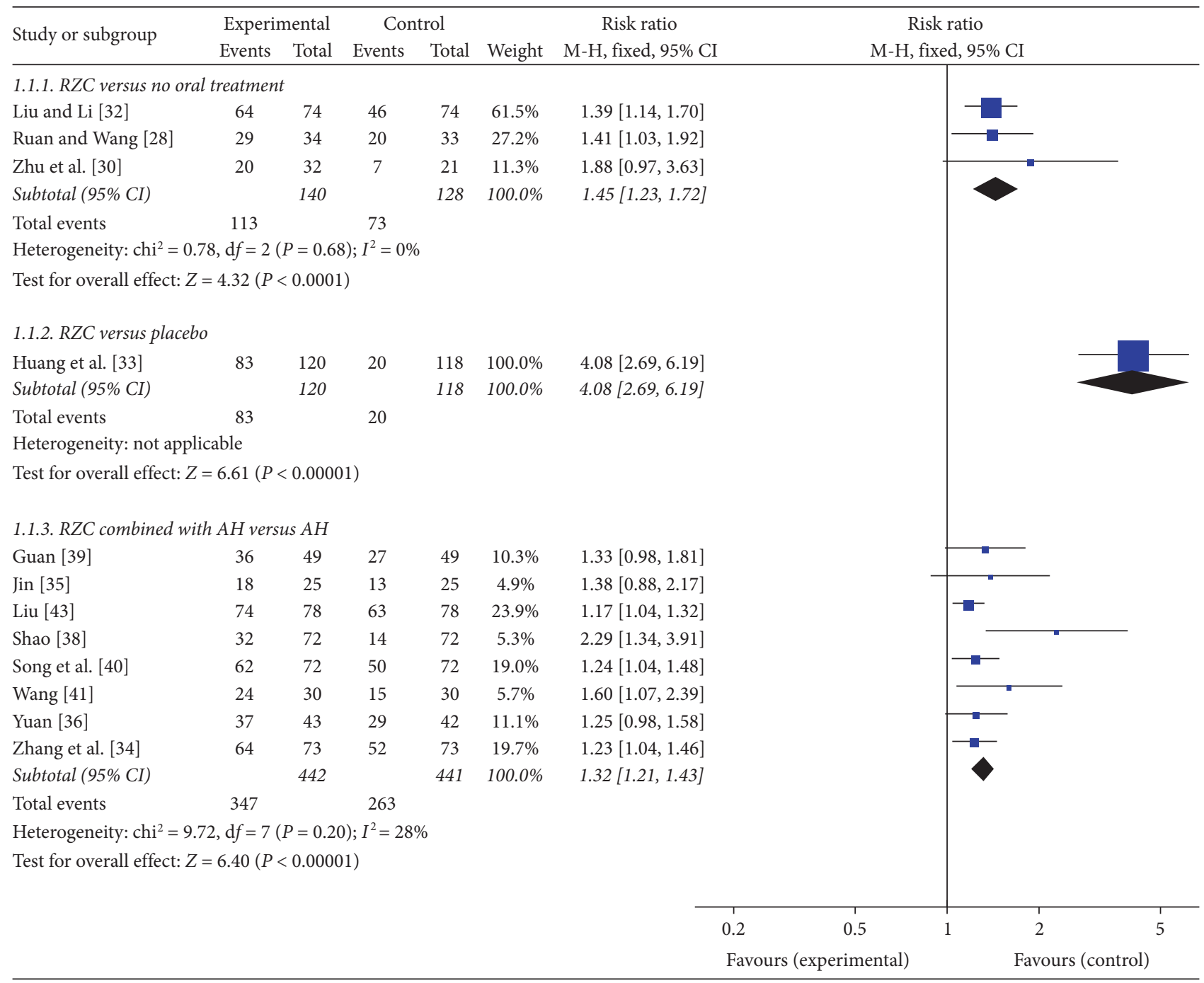

FIgURE 3: Forest plot of Runzao Zhiyang capsule on the TER.

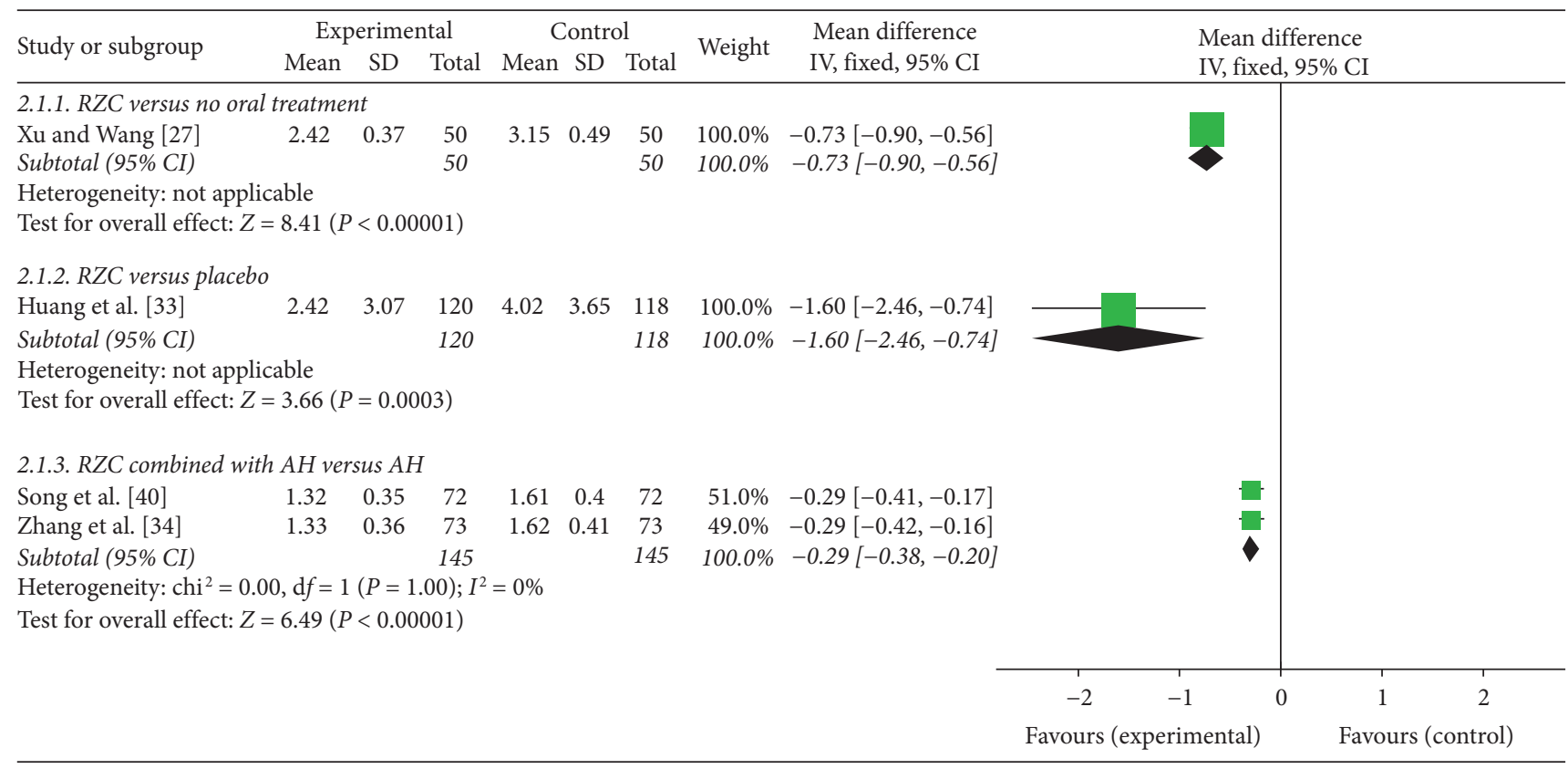




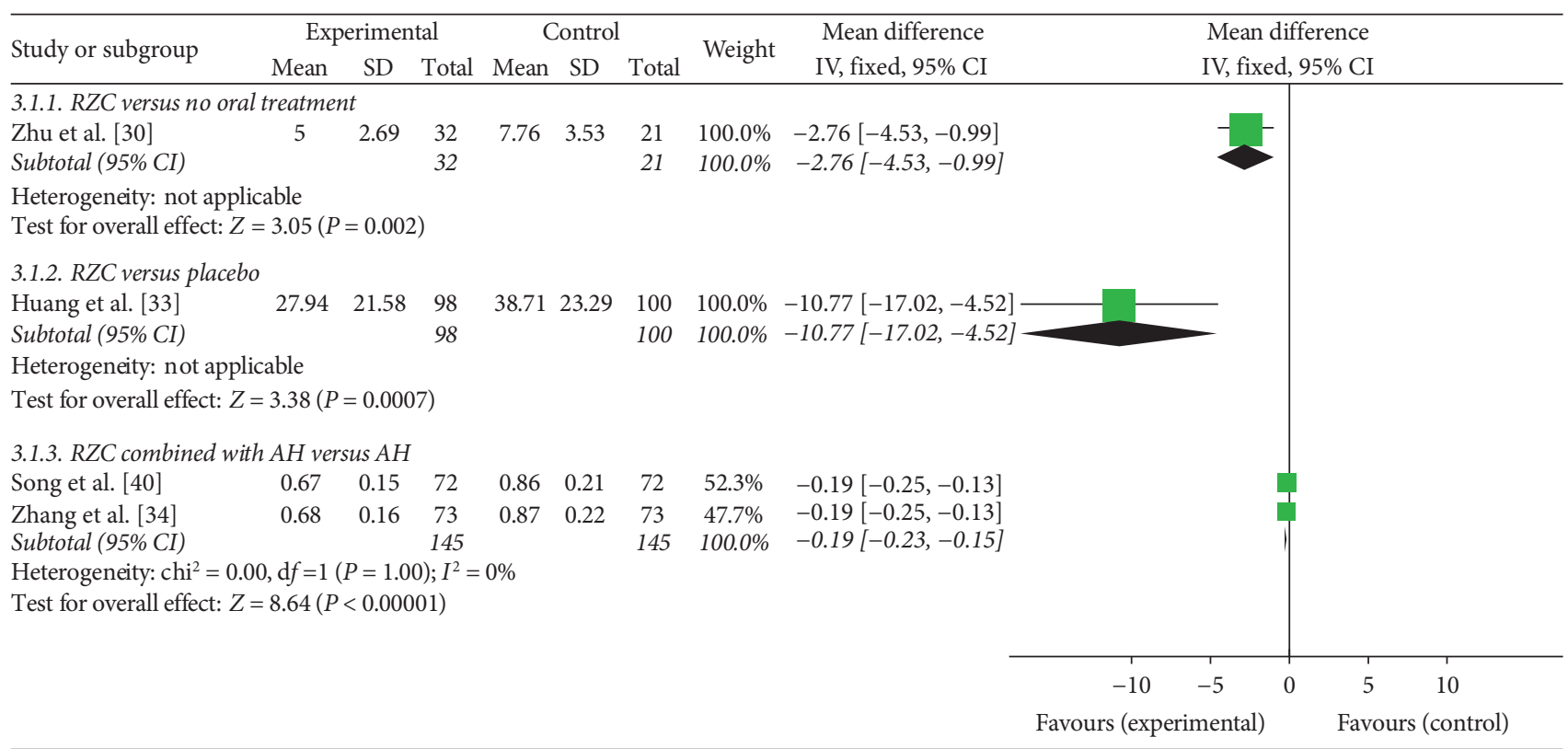

Figure 5: Forest plot of Runzao Zhiyang capsule on the VAS score.

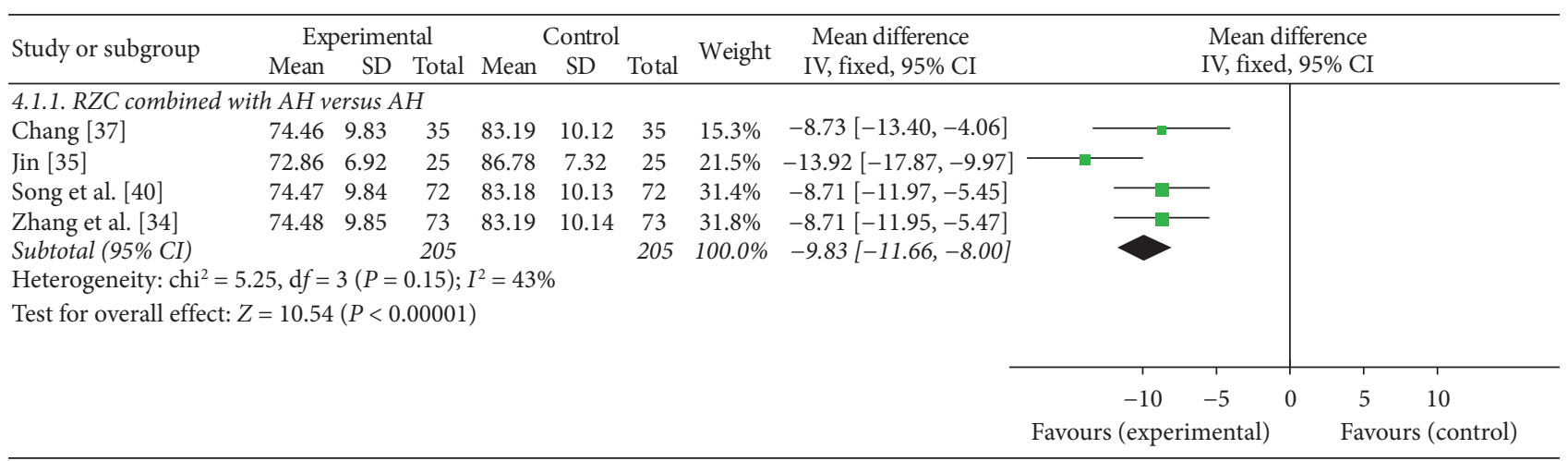

Figure 6: Forest plot of Runzao Zhiyang capsule on the serum total IgE level.

combined with AH could significantly improve TER and reduce EASI score, suggesting that $\mathrm{RZC}$ is also effective as an add-on treatment to $\mathrm{AH}$ and topical medicine. Therefore, the combination of $\mathrm{RZC}, \mathrm{AH}$, and topical medicine may provide a new treatment for refractory chronic eczema.

Itch is a defining symptom of eczema. It leads to scratching, resulting in more inflammation of skin, and a vicious "itch-scratch" circle is initiated. The pathophysiology of itch in eczema is not fully understood. Besides mast cellhistamines axis, basophiles are involved in acute itch flares, and many cytokines have also been identified, such as IL-4, IL-13, and substance P $[48,49]$. The current study showed that the VAS score of the RZC group reduced significantly compared with the placebo group and the no oral treatment group, indicating that RZC as an oral CHM is able to relieve itch effectively. In addition, the VAS score of the RZC combined with $\mathrm{AH}$ group was significantly lower than that of the AH group, suggesting that RZC could alleviate pruritus though histamine-independent pathways. The possible mechanism may be attributed to the fact that RZC could suppress the production of some inflammatory cytokines, such as IL-4 and IL-13. Therefore, RZC is effective as an add-on treatment for relieving itch.

In addition, some laboratory abnormalities can be found in the majority of eczema patients, such as serum total IgE level. IgE is a key molecule which can activate mast cells and basophils in allergic inflammation, and increased serum total IgE level is significantly correlated with the disease severity [50]. The current study showed that the serum total IgE level of the RZC combined with AH group was significantly lower than that of the AH group, which was also consistent with the change of EASI score. The result may be related to the effect of RZC on decreasing the level of IL-4 in serum.

In terms of safety, the current study showed that gastrointestinal diseases were the common adverse events of 


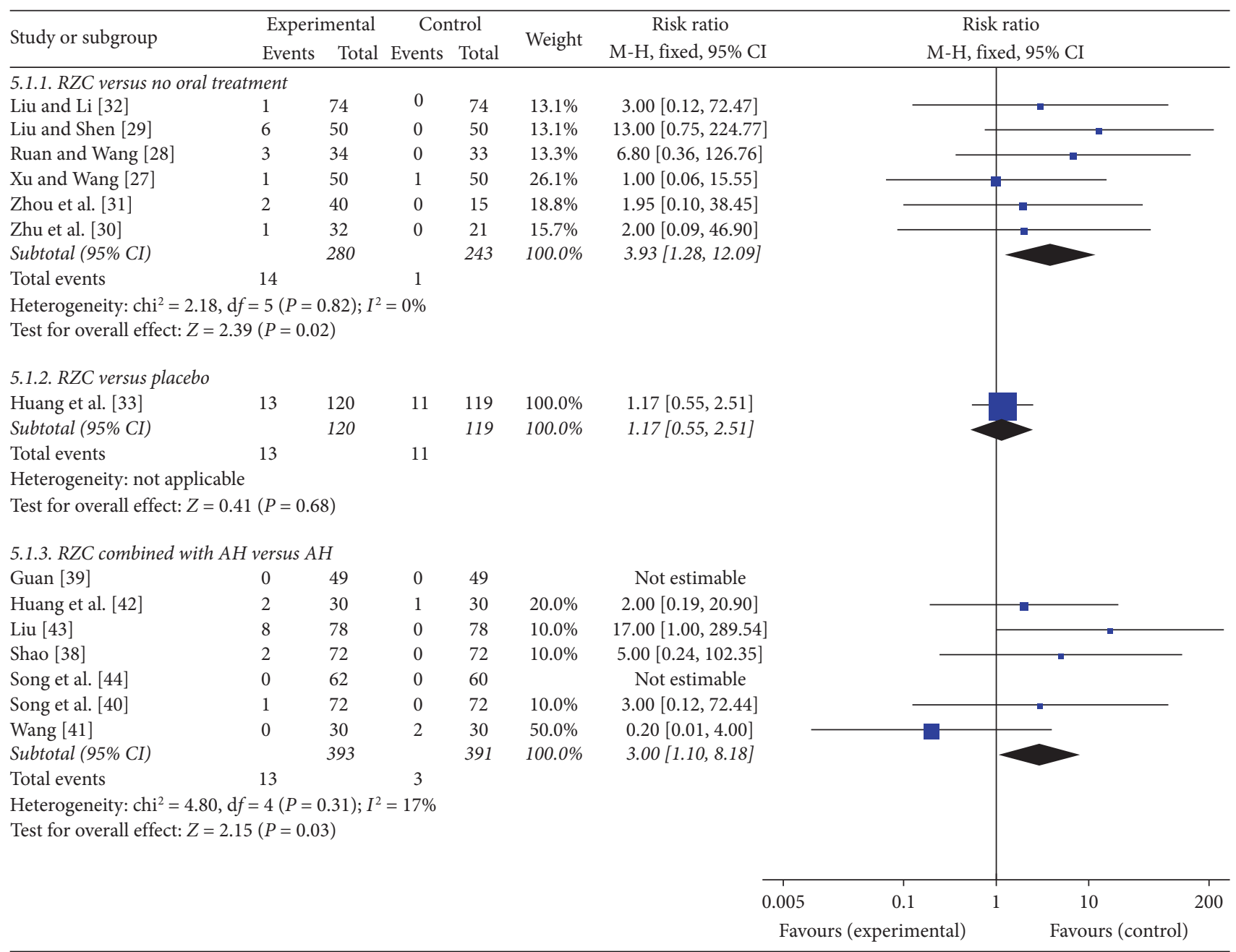

FIGURE 7: Forest plot of Runzao Zhiyang capsule on the gastrointestinal adverse events.

RZC, such as stomach discomfort and diarrhea, and gastrointestinal adverse events occurred more frequently in patients treated with RZC. Because the symptoms were mild and tolerable, there is no need to discontinue the treatment, and the symptoms could disappear spontaneously after drug withdrawal. On the other hand, laboratory examination is an important aspect of the drug safety. According to the six included studies, the majority of patients in the RZC group had normal liver and renal functions, while a few patients experienced a slight increase of liver transaminases, and the incidences of abnormal liver function between the RZC group and the placebo group were not statistically different [33]. In Chinese literature databases, five cases of liver injury during the treatment of RZC were reported, and the authors claimed the liver dysfunction may be related to Polygoni Multiflori Radix, an ingredient of RZC [51-54]. Multiple factors, such as genetic susceptibility and drug dosage, are involved in the mechanism of Polygoni Multiflori Radixinduced liver injury $[55,56]$. The correlation between RZC and liver injury is still uncertain and more clinical studies are needed. Therefore, administration of routine dose of RZC in the short time is safe, and regular examination of liver and renal function during the treatment can eliminate the occurrence of serious adverse events.

There are some limitations in this meta-analysis. First, the methodological quality of included studies is suboptimal. Most studies did not provide the information on the methods of randomization and blindness, which could reduce the reliability of results. Secondly, the number of the included studies was limited, and the sample sizes were small. Thirdly, the follow-up of the included studies is short. The efficacy and safety of the long-term use of RZC require a larger number of longer follow-up studies in the future. Finally, the conclusions on RZC for chronic eczema could not be generalized to children and other countries. In this study, few participants were children and all included studies were conducted in China.

\section{Conclusion}

In summary, RZC as an add-on therapy to conventional therapy has a good effect on chronic eczema in reducing skin lesion, relieving pruritus, and decreasing serum total IgE level. There does not appear to be any severe side effects from 
short-term use of RZC, and regular detection of liver and renal function is necessary in case of serious adverse events. However, because the methodological quality of the included studies is suboptimal, more large-scale and highquality studies are needed to confirm the current results in the future.

\section{Data Availability}

The data used to support this study are included within this article.

\section{Conflicts of Interest}

The authors declare that there are no conflicts of interest regarding the publication of this paper.

\section{Authors' Contributions}

ML and LFL conceived and designed the study. ML and BYZ conducted the literature search and study selection, extracted the data, and evaluated the quality of studies. LHZ verified the literature search, data extraction, and quality assessment. ML and BYZ analyzed the data and performed the statistical analysis. ML wrote the manuscript. LFL revised the manuscript. All authors read and approved the final manuscript.

\section{Supplementary Materials}

Supplementary Table 1: PRISMA checklist. (Supplementary Materials)

\section{References}

[1] S. G. O. Johansson, T. Bieber, R. Dahl et al., "Revised nomenclature for allergy for global use: report of the nomenclature review committee of the World Allergy Organization, october 2003," Journal of Allergy and Clinical Immunology, vol. 113, no. 5, pp. 832-836, 2004.

[2] A. Wollenberg, S. Barbarot, T. Bieber et al., "Consensus-based European guidelines for treatment of atopic eczema (atopic dermatitis) in adults and children: part I," Journal of the European Academy of Dermatology and Venereology, vol. 32, no. 5, pp. 657-682, 2018.

[3] S. Nutten, "Atopic dermatitis: global epidemiology and risk factors," Annals of Nutrition and Metabolism, vol. 66, no. 1, pp. 8-16, 2015.

[4] Y. Guo, P. Li, J. Tang et al., "Prevalence of atopic dermatitis in Chinese children aged 1-7 ys," Scientific Reports, vol. 6, Article ID 29751, 2016.

[5] F. Xu, S. Yan, F. Li et al., "Prevalence of childhood atopic dermatitis: an urban and rural community-based study in Shanghai, China," PLoS One, vol. 7, no. 5, Article ID e36174, 2012.

[6] A. T. M. Rønnstad, A. S. Halling-Overgaard, C. R. Hamann, L. Skov, A. Egeberg, and J. P. Thyssen, "Association of atopic dermatitis with depression, anxiety, and suicidal ideation in children and adults: a systematic review and meta-analysis," Journal of the American Academy of Dermatology, vol. 79, no. 3, pp. 448-456, 2018.
[7] Y. Schonmann, K. E. Mansfield, J. F. Hayes et al., "Atopic eczema in adulthood and risk of depression and anxiety: a population-based cohort study," The Journal of Allergy and Clinical Immunology: In Practice, vol. 8, no. 1, pp. 248-257, 2020.

[8] T. F. Tsai, M. Rajagopalan, C. Y. Chu et al., "Burden of atopic dermatitis in Asia," The Journal of Dermatology, vol. 46, no. 10, pp. 825-834, 2019.

[9] I. Katayama, M. Aihara, Y. Ohya et al., "Japanese guidelines for atopic dermatitis 2017," Allergology International, vol. 66, no. 2, pp. 230-247, 2017.

[10] W. Xu, Y. Li, M. Ju et al., "A multicenter, randomized, doubleblind, placebo-controlled study of compound Glycyrrhizin capsules combined with a topical corticosteroid in adults with chronic eczema," Evidence-Based Complementary and Alternative Medicine, vol. 2020, Article ID 6127327, 9 pages, 2020.

[11] M. Rahnar, A. Zarei, M. Ranjbar, M. Tabarrai, and L. Shirbeigi, "Etiology, clinical manifestation and natural treatment of psoriasis from the perspective of Persian medicine," Traditional and Integrative Medicine, vol. 5, no. 4, pp. 221-229, 2020.

[12] M. Meysami, M. H. Hashempur, M. Kamalinejad, and M. Emtiazy, "Efficacy of short term topical Malva sylvestris L. cream in pediatric patients with atopic dermatitis: a randomized double-blind placebo controlled clinical trial," Endocrine, Metabolic \& Immune Disorders-Drug Targets, vol. 20, 2020.

[13] C.-C. Lee, Y.-L. Lee, C.-N. Wang et al., "Polygonum multiflorum decreases airway allergic symptoms in a murine model of asthma," The American Journal of Chinese Medicine, vol. 44, no. 1, pp. 133-147, 2016.

[14] K.-H. Kang, K.-H. Lee, H.-M. Yoon, K.-J. Jang, C.-H. Song, and C.-H. Kim, "Rehmannia glutinosa pharmacopuncture solution regulates functional activation, FceRI expression, and signaling events in mast cells," Journal of Pharmacopuncture, vol. 15, no. 4, pp. 32-41, 2012.

[15] Y.-Y. Sung, T. Yoon, J. Y. Jang, S.-J. Park, and H. K. Kim, "Topical application of Rehmannia glutinosa extract inhibits mite allergen-induced atopic dermatitis in NC/Nga mice," Journal of Ethnopharmacology, vol. 134, no. 1, pp. 37-44, 2011.

[16] A. M. De Oliveira, S. Mesquita Mda, G. C. da Silva et al., "Evaluation of toxicity and antimicrobial activity of an ethanolic extract from leaves of Morus alba L. (Moraceae)," Evidence-Based Complementary and Alternative Medicine, vol. 2015, Article ID 513978, 7 pages, 2015.

[17] E. Park, S.-M. Lee, J. e. Lee, and J.-H. Kim, “Anti-inflammatory activity of mulberry leaf extract through inhibition of NF- $\kappa$ B," Journal of Functional Foods, vol. 5, no. 1, pp. 178-186, 2013.

[18] H. Kim, M. R. Lee, G. S. Lee, W. G. An, and S. I. Cho, "Effect of Sophora flavescens aiton extract on degranulation of mast cells and contact dermatitis induced by dinitrofluorobenzene in mice," Journal of Ethnopharmacology, vol. 142, no. 1, pp. 253-258, 2012.

[19] T. Yamaguchi-Miyamoto, T. Kawasuji, Y. Kuraishi, and H. Suzuki, "Antipruritic effects of Sophora flavescens on acute and chronic itch-related responses in mice," Biological and Pharmaceutical Bulletin, vol. 26, no. 5, pp. 722-724, 2003.

[20] X. Luo, L.-L. Li, S.-S. Zhang et al., "Therapeutic effects of total coumarins from urtica dentata hand on collagen-induced arthritis in Balb/c mice," Journal of Ethnopharmacology, vol. 138, no. 2, pp. 523-529, 2011. 
[21] J. Liu, "The observation of effect on Runzao zhiyang combined with levocetirizine for chronic eczema," Chinese Journal of Dermatovenereology, vol. 22, no. 6, pp. 3-4, 2008.

[22] T. Ma, Y. Chai, S. Li et al., "Efficacy and safety of Qinzhuliangxue decoction for treating atopic eczema: a randomized controlled trial," Annals of Palliative Medicine, vol. 9, no. 3, pp. $870-882,2020$.

[23] A. Liberati, D. G. Altman, J. Tetzlaff et al., "The PRISMA statement for reporting systematic reviews and meta-analyses of studies that evaluate health care interventions: explanation and elaboration," Journal of Clinical Epidemiology, vol. 62, no. 10, pp. 1-34, 2009.

[24] J. M. Hanifin, M. Thurston, M. Omoto et al., “The eczema area and severity index (EASI): assessment of reliability in atopic dermatitis," Experimental Dermatology, vol. 10, no. 1, pp. 11-18, 2001.

[25] A. Reich, M. Heisig, N. Phan et al., "Visual Analogue scale: evaluation of the instrument for the assessment of pruritus," Acta Dermato Venereologica, vol. 92, no. 5, pp. 497-501, 2012.

[26] J. P. T. Higgins, D. G. Altman, P. C. Gøtzsche et al., "The cochrane collaboration's tool for assessing risk of bias in randomised trials," BMJ, vol. 343, no. 2, Article ID d5928, 2011.

[27] B. Xu and M. Wang, "Clinical observation of Runzao Zhiyang capsules combined with hydrocortisone butyrate in the treatment of chronic eczema," Drugs and Clinic, vol. 34, no. 11, pp. 3367-3370, 2019.

[28] H. P. Ruan and L. Wang, "Mometasone furoate cream combined with Runzao zhiyang capsule for 34 cases of neurodermatitis," Modern Journal of Integrated Traditional Chinese and Western Medicine, vol. 20, no. 15, p. 1887, 2011.

[29] F. Y. Liu and Z. X. Shen, "Runzaozhiyang capsule combined with dexamethasone oitment for the treatment of neurodermatitis," Zhejiang Journal of Integrated Traditional Chinese and Western Medicine, vol. 21, no. 5, pp. 344-345, 2011.

[30] X. H. Zhu, Y. S. Yang, and J. H. Xu, "Observation of the effect of Runzao Zhiyang capsule for treating mild to moderated atopic dermatitis," Chinese Journal of Dermatovenereology, vol. 24, no. 1, pp. 38-39, 2010.

[31] H. B. Zhou, Y. Z. Ji, and L. Geng, "The effect of Runzaozhiyang capsule combined with hydrocortisone and urea cream for the treatment of neurodermatitis," Chinese Journal of Dermatovenereology, vol. 23, no. 7, pp. 457-458, 2009.

[32] H. X. Liu and H. W. Li, "Clinical observation of Runzao Zhiyang capsule for the treatment of chronic eczema and neurodermatitis," China Journal of Leprosy and Skin Diseases, vol. 25, no. 6, pp. 475-476, 2009.

[33] D. Huang, K. Chen, F.-R. Zhang et al., "Efficacy and safety of Run Zao Zhi Yang capsule on chronic eczema: a multiplecenter, randomized, double-blind, placebo-controlled clinical study," Journal of Dermatological Treatment, vol. 30, no. 7, pp. 677-684, 2019.

[34] H. C. Zhang, Y. P. Luo, and A. T. Liu, "The effect of Runzao Zhiyang capsules on the treatment of chronic eczema and its influence on the level of EOS and serum IgE in peripheral blood," China Modern Medicine, vol. 25, no. 18, pp. 45-48, 2018.

[35] X. Jin, "The effect of Run Zao Zhi Yang capsule combined with epinastine for chronic eczema," Psychological Doctor, vol. 24, no. 36, pp. 104-105, 2018.

[36] C. L. Yuan, "The efficacy of Runzaozhiyang capsule combined with desloratadine tablet for neurodermatitis," Chinese Journal of Modern Drug Application, vol. 12, no. 14, pp. 162-163, 2018.
[37] G. W. Chang, "The effect of Run Zao Zhi Yang capsule combined with epinastine capsule on inflammatory factor and IgE in serum for chronic eczema," Journal of Dermatology and Venereology, vol. 40, no. 5, pp. 681-683, 2018.

[38] W. Shao, "Effect of Run Zao Zhi Yang capsule for the treatment of chronic eczema," Journal of Clinical Medical, vol. 4, no. 72, p. 14218, 2017.

[39] X. Guan, "The therapeutic effect of Runzaozhiyang capsule combined with mizolastine sustained release tablets in the treatment of chronic eczema," China Continuing Medical Education, vol. 8, no. 33, pp. 225-226, 2016.

[40] Y. Z. Song, R. C. Lu, H. C. Lu, and T. L. Xie, "The effect of Runzao Zhiyang capsules for chronic eczema and its influence on the level of EOS and serum IgE in peripheral blood," Modern Journal of Integrated Traditional Chinese and Western Medicine, vol. 25, no. 30, pp. 3390-3392, 2016.

[41] X. Wang, "Observation of Runzaozhiyang capsule combined with epinastine capsule in the treatment of chronic eczema," Sichuan Medical Journal, vol. 36, no. 7, pp. 1008-1010, 2015.

[42] S. M. Huang, W. J. Ou Yang, J. X. Zhou, and Q. R. Jiang, "Runzaozhiyang capsule combined with levocetirizine tablet and hydrocortisone butyrate cream for 30 cases of neurodermatitis," Fujian Journal of Traditional Chinese Medicine, vol. 46, no. 5, p. 65, 2015.

[43] D. Liu, "Clinical observation on treating chronic eczema with cetirizine tablets plus the Runzao Zhiyang capsule," Clinical Journal of Chinese Medicine, vol. 28, no. 6, pp. 86-87, 2014.

[44] F. J. Song, Y. H. Huang, R. Chen, F. F. Li, and G. W. Yin, "Observation of the effect of Runzao Zhiyang capsule combined with mizolastine sustained release tablets for the treatment of chronic eczema," Journal of Medical Forum, vol. 35, no. 2, pp. 129-130, 2014.

[45] F. Lu, "Clinical observation of Runzao Zhiyang capsule combined with hydrocortisone butyrate cream for the treatment of chronic eczema," The Medical Forum, vol. 23, no. 32, pp. 4709-4710, 2019.

[46] Q. J. Li and J. D. Ma, "Effect of Runzao Zhiyang capsule combined with loratadine on stress response and inflammatory response in chronic eczema," Shanxi Journal of Traditional Chinese Medicine, vol. 39, no. 10, pp. 1447-1449, 2018.

[47] H. Y. Hu, Y. X. Hu, C. S. Liu, X. T. Su, and H. Dai, "Effects of Runzao Zhiyang capsule on gut microbiota and inflammatory response in rats with psoriatic lesisons," Chinese Journal of Clinical Anatomy, vol. 38, no. 5, pp. 574-577, 2020.

[48] G. Yosipovitch, T. Berger, and M. S. Fassett, "Neuroimmune interactions in chronic itch of atopic dermatitis," Journal of the European Academy of Dermatology and Venereology, vol. 34, no. 2, pp. 239-250, 2020.

[49] F. Wang, A. M. Trier, F. Li et al., "A basophil-neuronal axis promotes itch," Cell, vol. 184, no. 2, pp. 422-440, 2021.

[50] Y. Hu, S. Liu, P. Liu, Z. Mu, and J. Zhang, "Clinical relevance of eosinophils, basophils, serum total IgE level, allergenspecific IgE, and clinical features in atopic dermatitis," Journal of Clinical Laboratory Analysis, vol. 34, no. 6, Article ID e23214, 2020.

[51] D. C. Wang and R. Zhou, "Liver injury induced by Runzao Zhiyang capsule: a case report and literature review," Chinese Journal of Clinical Rational Drug Use, vol. 11, no. 2, pp. 19-22, 2018.

[52] Y. Y. Liu, "A case of liver injury induced by oral Runzao Zhiyang capsule," Chinese Hepatology, vol. 25, no. 5, pp. 556-557, 2020. 
[53] L. L. Tian, T. R. Zhou, J. Wu, and D. Q. Zhu, "A case of liver injury induced by oral Runzao Zhiyang capsule," Shanghai Medical and Pharmaceutical Journal, vol. 38, no. 17, pp. 3537, 2017.

[54] X. Wang, M. Xie, and X. H. Yi, "Clinical pharmacists participating in pharmaceutical care of two patients with druginduced liver injury caused by Runzao Zhiyang capsule," Central South Pharmacy, vol. 17, no. 6, pp. 919-921, 2019.

[55] C. Li, T. Rao, X. Chen et al., "HLA-B*35:01 allele is a potential biomarker for predicting polygonum multiflorum-induced liver injury in humans," Hepatology, vol. 70, no. 1, pp. 346357, 2019.

[56] X.-H. Xia, Y.-Y. Yuan, and M. Liu, "The assessment of the chronic hepatotoxicity induced by polygoni Multiflori radix in rats: a pilot study by using untargeted metabolomics method," Journal of Ethnopharmacology, vol. 203, pp. 182190, 2017. 